\title{
Experimental Implementation of UFAD Regulation based on Robust Controlled Invariance*
}

\author{
Pierre-Jean Meyer ${ }^{\dagger \ddagger}$, Hosein Nazarpour ${ }^{\dagger}$, Antoine Girard ${ }^{\dagger}$ and Emmanuel Witrant ${ }^{\ddagger}$
}

\begin{abstract}
In this paper, we implement a temperature regulation strategy on a small-scale building equipped with UnderFloor Air Distribution (UFAD). Using a 0 -dimensional model of the temperature variations in the building identified from experimental measurements, this strategy is based on the criteria of Robust Controlled Invariance, which give a characterization of the limits for a robust controller to keep the state of the system in an interval. These criteria are defined for monotone systems with bounded disturbances and thus are independent of the chosen control strategy.
\end{abstract}

\section{INTRODUCTION}

Energy consumption in buildings (both commercial and residential) has reached up to $40 \%$ of the total energy use in developed countries and is rapidly increasing with the growing demand in comfort [1]. This fact motivates many countries to make energy savings in buildings a priority, as mentioned by the European Energy Performance of Buildings Directives [2], [3]. As a result, a substantial amount of work has been done in the past decades toward intelligent buildings [4]. This has been particularly the case for climate regulation in buildings, with research on modeling, simulation [5] and control [6] of Heating, Ventilating and Air Conditioning (HVAC) systems, to improve comfort and energy efficiency. On these matters, the UnderFloor Air Distribution (UFAD) solution has shown some interesting results compared to the more traditional ceiling ventilation [7].

Various paths have already been explored for the control of HVAC systems in intelligent buildings. When the focus is mainly on control, numerous feedback strategies have been devised, based on simple PID or On/Off control, more robust controllers with the $\mathcal{H}_{\infty}$ approach [8], or non-linear approaches [9]. For more energy-efficient controllers, we can look for the optimal tradeoff between comfort and energy saving [10], a model-predictive strategy [11], or a fuzzy logic controller [12].

In this paper we consider the notion of Robust Controlled Invariance which characterizes whether a controller can maintain the state of the system in an interval for any value of the disturbances. With the monotonicity property [13], intuitive for thermal systems, we obtain simple new criteria on Robust Controlled Invariance. The goal of this paper is to use these criteria to implement a temperature regulation

\footnotetext{
*This work was partly supported by a PhD scholarship and the research project COHYBA funded by Région Rhône-Alpes.

${ }^{\dagger}$ Univ. Grenoble Alpes, LJK, F-38000 Grenoble, France:

\{Pierre-Jean. Meyer, Antoine.Girard\}@imag.fr nazarpur@gmail.com

¥Univ. Grenoble Alpes, GIPSA-Lab, F-38000 Grenoble, France: Emmanuel.Witrantegipsa-lab.grenoble-inp.fr
}

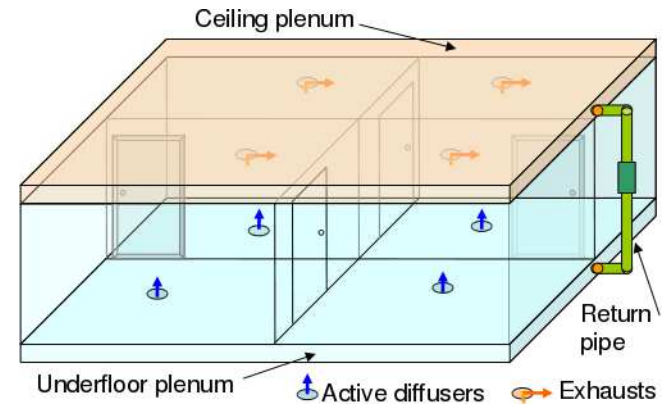

Fig. 1. 4-room flat with UFAD

on a small-scale UFAD flat. We should note that our focus is not on the efficiency of a controller but simply on checking if the invariance can be achieved in the chosen interval. For a deeper discussion on the monotonicity of our system and the results involving the Robust Controlled Invariance, the reader is referred to the theoretical counterpart of this paper in $[14]^{1}$.

The paper is organized as follows. In Section II, we describe the 0-dimensional UFAD model from [8], which is then identified with a small-scale experiment of a flat to match its behavior. In Section III, the monotonicity property is presented and analyzed for our 0D model, followed by the introduction of the Robust Controlled Invariance. The final Section IV gives an application of the obtained control criteria on the experiment and a comparison between these results and the identified model.

\section{Modeling}

The system considered is a small-scale flat equipped with UnderFloor Air Distribution (UFAD), as sketched in Fig. 1 and pictured in Fig. 2. The cooling of the room temperatures is done as follows. Peltier coolers are used to decrease the temperature of the air in the underfloor plenum, which is sent into each room of the building using the controlled fans. The excess of air is pushed into the ceiling plenum through exhausts in the fake ceiling and then sent back to the underfloor through a return pipe outside of the flat.

There are two components in the control problem. At the building level, we control the air recirculation with the speed of the fan in the return pipe and the temperature in the underfloor plenum by acting on the Peltier coolers. At the room level, we use the fans in the underfloor separately to

\footnotetext{
${ }^{1}$ An extended version of this paper containing the proofs is available at http://hal.archives-ouvertes.fr/docs/00/85/87/15/ $\mathrm{PDF} /$ Controllability.pdf
} 


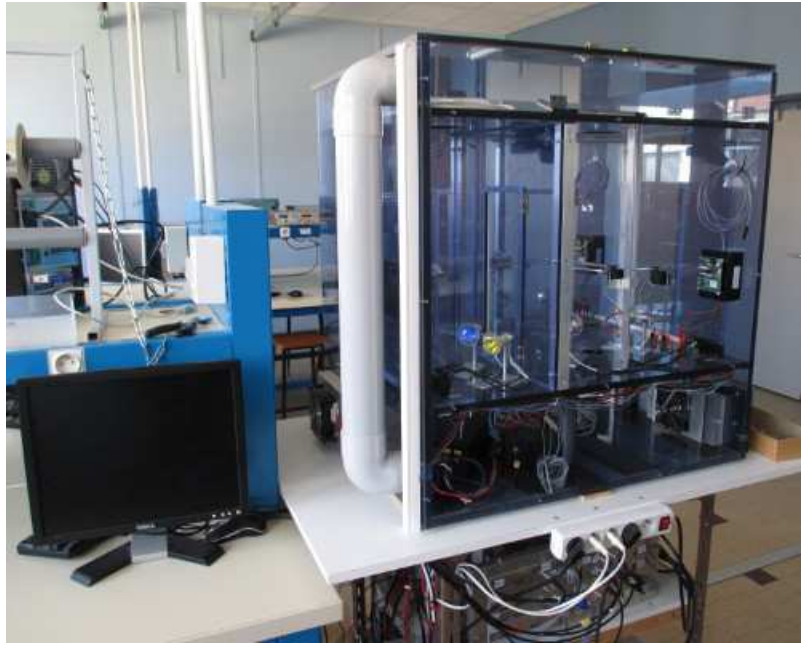

Fig. 2. Small-scale experiment of a flat with UFAD regulation ${ }^{2}$

obtain a decentralized control of each room temperature. In this paper, the controls at the building level are assumed to be set and our focus is on achieving separate climate regulation using decentralized control.

\section{A. Theoretical model}

We consider the following 0-dimensional model:

$$
\begin{aligned}
\forall i, \frac{d T_{i}}{d t}= & \sum_{j \in \mathcal{N}_{i}} a_{i, j}\left(T_{j}-T_{i}\right)+b_{i} u_{i}\left(T_{u}-T_{i}\right) \\
& +\sum_{j \in \mathcal{N}_{i}} \delta_{d_{i j}} c_{i, j} * h\left(T_{j}-T_{i}\right)+\delta_{s_{i}} d_{i}\left(T_{s_{i}}^{4}-T_{i}^{4}\right)
\end{aligned}
$$

with all constants $a, b, c, d$ being positive. A detailed description on establishing this model is given in [8] and general information on the heat transfers involved in (1) can be found in the following books on the design of HVAC systems [15], [16]. This model is obtained by combining energy conservation (the first law of thermodynamics) and mass conservation in each room $i$ of the building. It is assumed that: the temperature of air is uniform in each room; the air is incompressible; the potential and kinetic energies of air can be neglected due to its reduced mass and speed. Here, we review some of the hypotheses used to derive (1) in [8] in order to capture the heat transfers observed in the experiment.

The first heat transfer in (1) corresponds to the conduction between the considered room $i$ and the neighbor rooms (or the outside) $j \in \mathcal{N}_{i}$, through the PVC walls. The second term is linked to the mass flow rate $u_{i}$ forced by the fan from the underfloor (of temperature $T_{u}$ ) into room $i$. The command over the fan speed, and by extension the induced air flows $u_{i}$, are the control inputs of our system. On the second line of (1) are the disturbances: the flow going through open doors and the radiation from heat sources (human body, computer, $\ldots)$. The booleans $\delta_{d_{i j}}$ and $\delta_{s_{i}}$ describe the discrete state

\footnotetext{
${ }^{2}$ Built at the physics department (UFR PhITEM) of University Joseph Fourier, Grenoble, France.
}

of these disturbances: they are equal to 1 when the door between rooms $i$ and $j$ is open and when a heat source is active in room $i$, respectively. The function $h$ introduced in the term for the doors is defined by:

$$
h(x)= \begin{cases}0 & \text { if } x \leq 0 \\ x^{3 / 2} & \text { if } x>0,\end{cases}
$$

and it comes from the removal, using the mass conservation, of the flow going into the ceiling. As a result, the door heat transfer only appears in the equation of the coldest of the rooms connected by the door. Finally, our experiment has lamps in each room included as the radiating heat sources of temperature $T_{s_{i}}$.

In this paper, we write the system dynamics as

$$
\frac{d T}{d t}=f(T, u, w, \delta),
$$

where the four-dimensional vector field $f$ depends on the state $T=\left[T_{1}, T_{2}, T_{3}, T_{4}\right]$, the control input $u=$ $\left[u_{1}, u_{2}, u_{3}, u_{4}\right]$, the boolean disturbances (doors and sources) $\delta$, and the exogenous input vector $w$ corresponding to the other temperatures $\left(T_{u}\right.$ for the underfloor, $T_{c}$ for the ceiling, $T_{o}$ for the outside). Each component $f_{i}$ of $f$ follows (1).

\section{B. Process identification}

The theoretical model (1) is adapted to the measured behavior of our experiment with the following identification strategy. We run several experiments on the flat to capture the main behaviors modeled in (1). In these tests, the outside temperature $T_{o}$ is varying around $30^{\circ} \mathrm{C}$ and the underfloor temperature $T_{u}$ is regulated at $17^{\circ} \mathrm{C}$ using a PID controller. Our experiments are quickly summarized as follows, with the desired physical phenomena.

- To observe the heat radiation, in each room, we turn on a lamp, wait for an equilibrium to be reached, then turn the lamp off;

- for the air flow coming from the underfloor, in each room, we turn on a fan, wait for an equilibrium to be reached, then turn the fan off;

- for the air flow through a door, we heat one of the neighbor room with a lamp to create a temperature gradient, then open the door;

- similarly to the previous test, we create a temperature gradient at the door by cooling down a neighbor room using a fan, then opening the door. This test is also added to check the influence of the fans on the direction of the air flow through the door;

- in each room, we alternatively turn on and off both fans and lamps to generate a data set that is sufficiently representative of the different operating conditions.

All these tests aim to quantify heat transfers due to heat radiation and the exchange of air flows, as well as the conduction in the walls.

The control of the experiment (fans, lamps, doors) and the measurements are done using LabVIEW ${ }^{\mathrm{TM}}$. The data are then processed in MATLAB ${ }^{\circledR}$. In our problem we have 

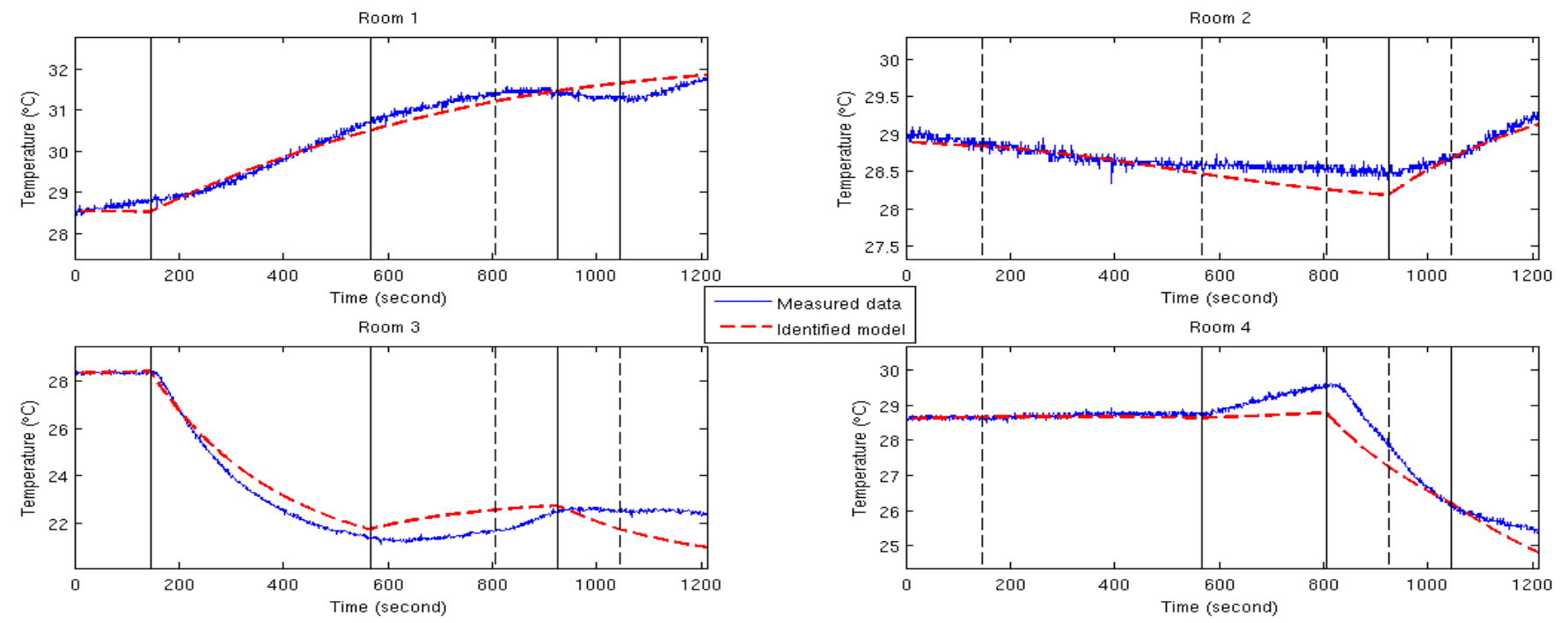

Fig. 3. Comparison between the identified model (dashed, red) and the verification data set (noisy, blue), with the transitions (vertical lines: solid when linked to the room, dashed otherwise)

40 unknown parameters, with 10 for each room: 5 for the conduction (underfloor, ceiling, outside, and 2 neighbor rooms), 1 for the fan, 1 for each door, and 2 for the heat source (emissivity and temperature). We solve this optimization problem with a least-squares algorithm initialized with a set of values based on known physical parameters and observations. We run this optimization on 45652 algebraic relationships obtained from the discretized system, representing the 57079 data points from the above experiments.

\section{Model evaluation}

The identified model is evaluated on experimental scenarios not included in the identification data set. An example of such scenario is described below, where we start with all lamps and fans off and all doors closed:

$$
\begin{array}{ll}
t=150 \mathrm{~s}, & \text { lamp } 1 \text { and fan } 3 \text { on; } \\
t=570 \mathrm{~s}, & \text { lamp } 3 \text { on, door } 1-4 \text { open; } \\
t=810 \mathrm{~s}, & \text { fan } 4 \text { on; } \\
t=930 \mathrm{~s}, & \text { lamp } 3 \text { off, door } 1-2 \text { open; } \\
t=1050 \mathrm{~s}, & \text { door } 1-4 \text { closed. }
\end{array}
$$

This data set gathers the main situations that can happen in the building: conduction alone (rooms 2 and 4); a lamp (room 1); a fan (room 3); an open door (rooms 2 and 4); a lamp and a fan (room 3); a lamp and an open door (room 1 ); a fan and an open door (room 4).

The data measured during this experiment (noisy, blue) is displayed and compared to the model (dashed, red) in Fig. 3. The vertical lines correspond to the transitions described in the previous paragraph: solid when the switched element has a direct link to the corresponding room and dashed otherwise. For the most part, we can see in Fig. 3 that the model fits reasonably well the data. For this data set of 1211 points, the mean squared error between the data and the model is $0.18^{\circ} \mathrm{C}$, with a standard deviation of 0.42 .

Even though this data set is representative of the typical phenomena observed in the building, it is only a sample of all the possible combinations between doors, fans and lamps to be verified. In a more thorough verification, we found several limitations to our model, some of which are visible in Fig. 3. First, if we look at the behavior in room 1 (top left of Fig. 3), the temperature of the model keeps increasing without any influence (i.e. cold air inflow) from the open doors with rooms 2 or 4 , since room 1 has the highest temperature (0D hypothesis that the air flows from the hot volume toward the cold one, due to pressure difference at constant density). In reality, we can see on the measured data that the door opening has some circulation effect on the hottest room. This is particularly visible between 810 and 1050 seconds, when the door between rooms 1 and 4 is open and the fan is running in room 4. This fan generates an air flow going in the opposite direction of the flow created by the pressure difference at the door. This behavior is not modeled in (1), and may become a problem when using the fans too fast. An extra term representing the turbulent diffusive mixing could be introduced if a refined model is required.

Another difference between the model and the data can be seen on the graph for room 3 (bottom left of Fig. 3). In this room, while the fan is running, the lamp is turned on at 570 seconds and turned off at 930 seconds. The difference between the model and the data in that case can be explained by the fact that the fan forces convection on the lamp. Indeed, the moving air creates a new convective heat transfer between the hot lamp (even when it has been turned off) and the air.

Also, the identified model is based on the ON/OFF use of the fans. The air flow induced by the maximal fan voltage is thus part of the parameters to identify and is included in the obtained values for $b_{i}$ in (1). In the next sections, when we apply a voltage between the extremal admissible values, we consider in the model that the air flow is linear to the voltage. To model the friction in the fans, we add a lower saturation of the air flow $\left(u_{i}=0\right)$ when the voltage is below a threshold. 


\section{Monotonicity And Robust Controlled INVARIANCE}

For rigorous definitions and proofs of the notions presented in this section, see the extended version of [14] available at the link given in page 1 .

\section{A. Monotonicity}

To obtain the controlled invariance proposed in the next section, the system needs to satisfy the monotonicity property. In [13], Angeli and Sontag define a monotone system as a system preserving partial orderings chosen for the state and the inputs. The cooperative systems, a subclass of the monotone systems but with a very similar definition, take their names from an interpretation which gives a clearer understanding of how such systems behave. A system is called cooperative because its variables reinforce each other, or more generally, "each pair of variables may affect each other in either positive or negative forms" [17].

This property is particularly easy to observe on thermal systems: if we take the example of the conduction $a_{i, j}\left(T_{j}-\right.$ $T_{i}$ ) (when considering room $i$ ), we naturally see that the hotter the neighbor $T_{j}$, the bigger the heat transfer toward room $i$. This comment can be reproduced for any of the heat transfers modeled in (1), whether $T_{j}$ represents a state or a temperature from $w$. In a similar way, for the discrete disturbances $\delta$ : if more $\delta_{d_{i j}}$ and $\delta_{s_{i}}$ are equal to 1 , we have more heat transfers going toward the considered room $i$, reinforcing the state $T_{i}$. This is natural for heat sources, since it is assumed that the source temperature is always hotter than the room temperature. In the case of the doors, remember that the heat transfer in (1) only appears in the coldest room. So having $\delta_{d_{i j}}=1$ with $T_{j}>T_{i}$ indeed reinforces $T_{i}$, while having no effect on $T_{j}$.

The variables $T, w$ and $\delta$ thus all affect the state $T$ in a positive way. In the case of our flat, we aim to cool down the four rooms, so the underfloor temperature is necessarily set to a value below any room temperature: $T_{u}<\min _{i}\left(T_{i}\right)$. Then, the heat transfer in room $i$ corresponding to the air flow forced by the fan is always negative $\left(b_{i} u_{i}\left(T_{u}-T_{i}\right)<0\right)$, resulting in a decrease of $T_{i}$. The control input $u$ thus affects the state $T$ in a negative way: increasing the air flow $u_{i}$ would decrease the room temperature $T_{i}$ faster.

The fact that the variables affect the state in different ways is not a problem: what matters is that each variable always has the same effect, which is indeed our case. According to the interpretation given in the first paragraph, our system is cooperative, and therefore monotone.

We can now address the initial definition of a monotone system, involving preservation of the partial orderings chosen for the state and the inputs. Let's consider two sets of variables $(T, u, w, \delta)$ and $\left(T^{\prime}, u^{\prime}, w^{\prime}, \delta^{\prime}\right)$ where $T$ is an initial state and $u, w, \delta$ are functions of time. We can choose partial orderings consistent with the above observations:

$$
\left\{\begin{aligned}
& T>T^{\prime} \\
\forall t>0, & u(t)<u(t)^{\prime} \\
\forall t>0, & w(t)>w(t)^{\prime} \\
\forall t>0, & \delta(t)>\delta(t)^{\prime} .
\end{aligned}\right.
$$

In that case, knowing that the system is monotone means that the trajectory of the system with $(T, u, w, \delta)$ stays at all time above the trajectory of the same system with $\left(T^{\prime}, u^{\prime}, w^{\prime}, \delta^{\prime}\right)$.

\section{B. Robust Controlled Invariance}

The Robust Controlled Invariance is the main notion used in the remaining of this paper. We aim to use the control input $u$ to keep the state in a chosen target interval $[\underline{T}, \bar{T}]$ for any external conditions $(w, \delta)$.

We consider that all the inputs of our system are bounded: either by physical constraints for the control inputs $\left(u_{i} \in\right.$ $\left.\left[0, \overline{u_{i}}\right]\right)$ and the disturbances $(\delta \in[0,1])$; or by a range obtained from observations: $w \in[\underline{w}, \bar{w}]$. With that assumption, we can use the monotonicity to simplify the conditions for Robust Controlled Invariance by considering the worst case for the external conditions ( $w$ and $\delta$ ) while taking the best control input $u$ to counteract the situation induced by the disturbances. If we take the example of the upper bound $\bar{T}$ of the interval, the worst situation of the disturbances is the hottest one ( $w=\bar{w}$ and $\delta=1)$ which tries to push the state above the interval. To compensate this effect, we choose the best ventilation $u=\bar{u}$ to cool down as much as possible. If in that situation we are able to decrease the temperature, it means that even in the hottest conditions, we can prevent the state from going over the upper bound $\bar{T}$.

We can now characterize the Robust Controlled Invariance using componentwise inequalities on the vector field.

Theorem 1: The system is Robust Controlled Invariant in $[\underline{T}, \bar{T}]$ if and only if

$$
\left\{\begin{array}{l}
f(\bar{T}, \bar{u}, \bar{w}, 1) \leq 0 \\
f(\underline{T}, 0, \underline{w}, 0) \geq 0 .
\end{array}\right.
$$

To simplify the notations in what follows, we define the subspaces $\mathcal{C}$ and $\mathcal{W}$ as the sets of admissible upper bound $\bar{T}$ and lower bound $\underline{T}$ respectively, so that the conditions in Theorem 1 can simply be written $\bar{T} \in \mathcal{C}$ and $\underline{T} \in \mathcal{W}$.

\section{ApPlication to the UFAD EXPERIMENT}

\section{A. Controller implementation}

When the state space is of dimension 2, we can easily obtain a graphical representation of the subspaces $\mathcal{C}$ and $\mathcal{W}$, which facilitates the choice of the target interval [14]. In our case, such a graphical representation is more complicated to obtain due to the 4 dimensions. Instead, we simply compute the extremal points of these subspaces (lower bound of $\mathcal{C}$ and upper bound of $\mathcal{W}$ ). These values would represent the minimal allowed $\bar{T}$ (lower bound of $\mathcal{C}$ ), and the maximal allowed $\underline{T}$ (upper bound of $\mathcal{W}$ ) in order to obtain the Robust Controlled Invariance. 
The characterization of the subspaces $\mathcal{C}$ and $\mathcal{W}$ (and therefore of the Robust Controlled Invariance) requires to consider that all the inputs of the system are bounded. The control input $u$ is clearly bounded between 0 and the air flow induced by the maximal voltage applied to the fans. The discrete disturbances in $\delta$ can only take two values: 0 or 1 . For the exogenous temperatures $w$, we take the intervals corresponding to observations in similar situations: both the ceiling and the outside temperatures are assumed to be varying between $27^{\circ} \mathrm{C}$ and $30^{\circ} \mathrm{C}$. For the underfloor temperature, we use a PID controller to maintain it at $17^{\circ} \mathrm{C}$. However, it has been observed that when all the fans are active, the Peltier coolers are not powerful enough to cool down the hot air coming from the ceiling. That is why we consider the following boundaries for the underfloor temperature: $T_{u} \in[17,21]$.

Knowing these boundaries, we can now obtain the lower bound of $\mathcal{C}: \min (\bar{T})=[26.1,26.2,29.0,28.1]$; and the upper bound of $\mathcal{W}: \max (\underline{T})=[26.2,26.3,26.4,26.5]$. The smallest Robust Controlled Invariant interval is wider for rooms 3 and 4 because room 3 is the smallest room and the heat radiated by the lamp in room 4 is more important than in other rooms. When we consider the hottest situation $(\mathcal{C})$, the heat radiation from the lamps thus has more impact on rooms 3 and 4 . For this control experiment, we choose a $3{ }^{\circ} \mathrm{C}$-wide target interval for each room, apart from room 3 for which we take it $4^{\circ} \mathrm{C}$-wide:

$$
\underline{T}=\left(\begin{array}{c}
26 \\
25 \\
26 \\
26
\end{array}\right) \quad \bar{T}=\left(\begin{array}{c}
29 \\
28 \\
30 \\
29
\end{array}\right)
$$

In Theorem 1, only one condition is implied on the controller: to use the maximal ventilation $\overline{u_{i}}$ when a room temperature reaches its upper bound $\overline{T_{i}}$ and to stop the fan when $T_{i}=T_{i}$. This leaves the choice of the control strategy relatively free to meet some performance specifications. In the scope of this paper, we do not aim to discuss the efficiency of a controller but simply to check the Robust Controlled Invariance with any controller. This is why we choose a simple linear controller, with saturations to satisfy the condition above. In the model described in (1), we consider that the control input is the mass flow rate $u$ sent by the fans. In reality, we do not have a direct control on the air flow, but only on the voltage $V$ applied to the fans. We consequently control the fan voltages in this Decentralized Linear Saturated (DLS) strategy.

Definition 1 (Decentralized Linear Saturated Controller):

$$
\forall i, \quad \begin{cases}T_{i} \leq \underline{T_{i}} & \Rightarrow V_{i}=0 \\ T_{i} \in\left[\underline{T_{i}}, \overline{T_{i}}\right] & \Rightarrow V_{i}\left(T_{i}\right)=\overline{V_{i}} * \frac{T_{i}-\underline{T_{i}}}{\overline{T_{i}}-\underline{T_{i}}} \\ T_{i} \geq \overline{T_{i}} & \Rightarrow V_{i}=\overline{V_{i}}\end{cases}
$$

As explained at the end of Section II-C, we only used the control input in ON/OFF mode until now. Indeed, since intuitively the air flow is monotone with respect to the voltage applied to the fan, our only concerns to ensure the invariance are the extremal values. However the controller from Definition 1 can assign to the fan any value in the voltage range $[0,6]$, so if we want to compare the measurements from the controlled experiment with the corresponding simulation we need to add to the model a function giving the relation between voltage and air flow of the fan. First, to model the frictions preventing the fan from working with a voltage below a threshold, we add a lower saturation $\left(u_{i}=0\right)$ for these small voltages. The observed voltages for the threshold in each room are: $[2,3.5,2.5,2.5]$. To keep the model as simple as possible for the remaining voltage range, we consider that the air flow is linear in the voltage, with the maximal air flow already obtained in the identification. The reality is that these two variables are not directly proportional, which can explain some of the variations between the measurements and the simulation in the results displayed in the next section (Fig. 4).

Using the DLS control strategy from Definition 1, we aim to regulate each temperature of the experiment in its target interval defined in (3). To ensure that the system encounters several disturbances, including both extremal conditions, we apply the following disturbance schedule, starting when all doors are closed and all lamps are off:

$$
\begin{array}{ll}
t=330 \mathrm{~s}, & \text { lamps } 2 \text { and } 3 \text { on; } \\
t=930 \mathrm{~s}, & \text { doors } 1-2 \text { and } 2-3 \text { open; } \\
t=1530 \mathrm{~s}, & \text { lamp } 4 \text { on, door } 3-4 \text { open; } \\
t=2010 \mathrm{~s}, & \text { lamp } 3 \text { off, doors } 2-3 \text { and } 3-4 \text { closed; } \\
t=2500 \mathrm{~s}, & \text { lamp } 4 \text { off, door } 4-1 \text { open; } \\
t=2910 \mathrm{~s}, & \text { lamp } 1 \text { on, door } 4-1 \text { closed; } \\
t=3510 \mathrm{~s}, & \text { all lamps on, all doors open; } \\
t=4350 \mathrm{~s}, & \text { all lamps off, all doors closed. }
\end{array}
$$

\section{B. Experimental results}

The results from that scenario are displayed (in blue) in Fig. 4. The horizontal black lines are the boundaries of the target interval given in (3). As in Fig. 3, the vertical lines represent the switching times in the schedule described above: solid lines when the switched component (door or lamp) is link to the room; dashed otherwise. In addition, the red dashed curves in Fig. 4 correspond to the simulation of this control using the identified model from Section II-B and the same exogenous inputs $w$ and disturbances $\delta$.

In room 3 (bottom left of Fig. 4), when the temperature increases the model is significantly higher than the measurements. This can be explained by the observed limitation of the model, described in Section II-C, when open doors and fans are associated. In the experiment of Fig. 4, room 3 has a higher temperature than its neighbors (2 and 4$)$. With the DLS controller from Definition 1, the fans are running most of the time. When the doors in room 3 are open (between 930 and 2100 seconds and between 3510 and 4350 seconds), the flows from the fans 2 and 4 will go in the opposite direction of the flows at the doors (supposed to go from the hot room 3 to the colder 2 and 4). This phenomenon, which is not modeled in (1), explains the differences between the model and the data in room 3 . 

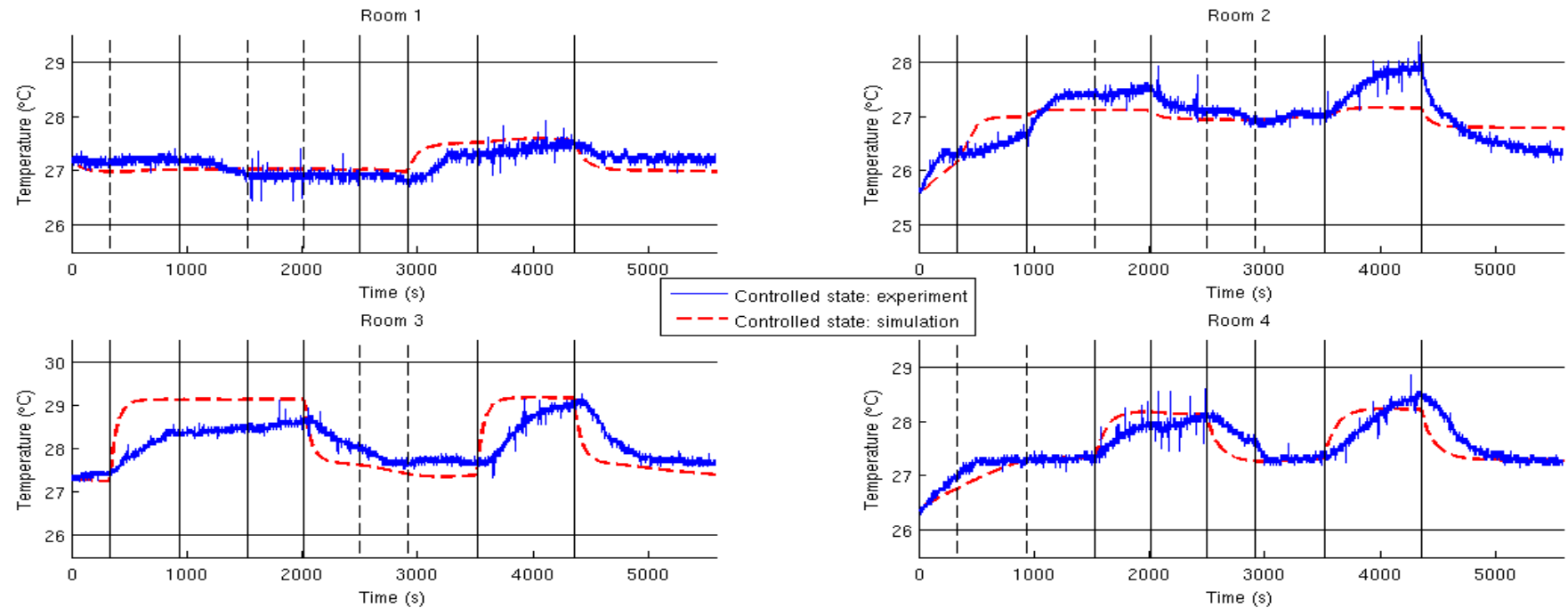

Fig. 4. Controlled state, using DLS controller, for the experiment (blue, noisy) and the simulation (red, dashed), with the transitions (vertical lines: solid when linked to the room, dashed otherwise)

The last observation we can do on the results in Fig. 4 is that the system is indeed invariant in the target interval chosen in (3). As previously listed in the disturbance schedule, both the hottest and the coldest situations are met for the discrete disturbances $\delta$. However, we can neither ensure that the extremal conditions of the exogenous inputs $w$ were met, nor that they were met at the same time as the extremal values of $\delta$. But we can note that these temperatures mostly intervene in the conduction which has a reduced effect on the system compared to the other heat transfers associated with the heat radiation of the air flows. We can thus almost claim (due to the model uncertainties) that the system is indeed Robust Controlled Invariant in the chosen target interval.

\section{CONCLUSION}

In this paper, we use a 0-dimensional model to describe the temperature dynamics in a building equipped with UnderFloor Air Distribution. Identification and verification methods are then used to fit the behavior of a smallscale experiment of an UFAD flat. The Robust Controlled Invariance is presented as the ability to maintain the state in a given target interval for any value of the disturbances and this result is greatly simplified by the monotonicity property satisfied by our model. Finally, the invariance criteria are computed using the identified model and confirmed in a control experiment on the small-scale UFAD flat.

The results on Robust Controlled Invariance presented in this paper can be generalized to any system provided it satisfies the monotonicity property and a local control property (each controlled input only affects one state variable). While we do not know a general category of systems satisfying the latter, we can find the monotonicity property in various fields such as molecular biology, chemical reactions, vehicle platooning, ... Furthermore, this approach does not depend on the feedback control strategy used in the Robust Controlled Invariant interval, which leaves a large degree of freedom for the performance specifications.

\section{REFERENCES}

[1] L. Perez-Lombard, J. Ortiz, and C. Pout, "A review on buildings energy consumption information," Energ. Buildings, vol. 40, no. 3, pp. 394 398, 2008.

[2] "Directive 2002/91/EC of the European parliament and of the council," Official Journal of the European Communities, December 2002.

[3] "Directive 2010/31/EU of the European parliament and of the council," Official Journal of the European Union, May 2010.

[4] J. K. W. Wong, H. Li, and S. W. Wang, "Intelligent building research: a review," Automat. Constr., vol. 14, no. 1, pp. 143-159, 2005.

[5] M. Trčka and J. L. M. Hensen, "Overview of HVAC system simulation," Automat. Constr., vol. 19, no. 2, pp. 93-99, 2010.

[6] H. Mirinejad, S. H. Sadati, M. Ghasemian, and H. Torab, "Control techniques in heating, ventilating and air conditioning systems," Journal of Computer Science, vol. 4, no. 9, pp. 777-783, 2008.

[7] F. S. Bauman and A. Daly, Underfloor air distribution (UFAD) design guide. ASHRAE, 2003.

[8] E. Witrant, P. Di Marco, P. Park, and C. Briat, "Limitations and performances of robust control over WSN: UFAD control in intelligent buildings," IMA J. Math. Control I., vol. 27, no. 4, pp. 527-543, 2010.

[9] B. Arguello-Serrano and M. Velez-Reyes, "Nonlinear control of a heating, ventilating, and air conditioning system with thermal load estimation," IEEE Trans. Contr. Syst. Technol., vol. 7, no. 1, pp. 5663, 1999.

[10] J. M. House and T. F. Smith, "Optimal control of building and HVAC systems," in Proc. ACC'95, vol. 6, 1995, pp. 4326-4330.

[11] F. Oldewurtel, A. Parisio, C. N. Jones, M. Morari, D. Gyalistras, M. Gwerder, V. Stauch, B. Lehmann, and K. Wirth, "Energy efficient building climate control using stochastic model predictive control and weather predictions," in Proc. ACC'10, 2010, pp. 5100-5105.

[12] M. Hamdi and G. Lachiver, "A fuzzy control system based on the human sensation of thermal comfort," in Proc. FUZZ-IEEE'98, vol. 1, 1998, pp. 487-492.

[13] D. Angeli and E. D. Sontag, "Monotone control systems," IEEE Trans. Automat. Contr., vol. 48, no. 10, pp. 1684-1698, 2003.

[14] P.-J. Meyer, A. Girard, and E. Witrant, "Controllability and invariance of monotone systems for robust ventilation automation in buildings," in Proc. IEEE CDC'13, 2013.

[15] G. J. Levermore, Building Energy Management Systems: Applications to Low-Energy HVAC and Natural Ventilation Control, 2nd ed. Taylor \& Francis, 2002.

[16] F. C. McQuiston, J. D. Parker, and J. D. Spitler, Heating, ventilating, and air conditioning: analysis and design, 6th ed. Wiley, 2010.

[17] D. Angeli and E. D. Sontag, "Interconnections of monotone systems with steady-state characteristics," in Optimal control, stabilization and nonsmooth analysis. Springer Berlin Heidelberg, 2004, pp. 135-154. 